\title{
Fair Pharma? Klinische Versuche: Verantwortung und Kontrolle
}

\section{Jacques Schiltknecht}

Herrn Dr. Etzel Gysling danke ich herzlich für die kritische Durchsicht des Manuskripts und für seine wertvollen Anregungen.

Eine Stellungnahme der forschenden pharmazeutischen Industrie der Schweiz wird in der nächsten SÄZ-Ausgabe veröffentlicht.
«Es ist die Pflicht des Arztes, in der medizinischen Forschung Leben, Gesundheit, Privatsphäre und Würde des Menschen zu schützen.»

(«Deklaration von Helsinki» des Weltärztebundes, 2000)

Anfang September 2013 gab die «Erklärung von Bern» (EvB) ein Sonderheft mit dem provokativen Titel «Menschliche Versuchskaninchen zum Schnäppchenpreis» heraus. Darin wurden viele Beispiele von ethisch nicht verantwortbaren Vorkommnissen bei der Durchführung klinischer Versuche durch Pharmafirmen angeprangert. Auf 23 Seiten werden mit guten Belegen, Statistiken und Grafiken gefährliche Trends aufgezeigt, die mir bisher nicht bewusst waren.

Beschämt musste ich feststellen, dass ich in den 40 Jahren meiner Praxistätigkeit als Allgemeinarzt nicht oft an die Menschen gedacht habe, die ihren Körper für klinische Versuche zur Verfügung stellen. Aufrüttelnde Artikel, z.B. von Etzel Gysling in der «pharma-kritik», verklangen bald im Strudel des Praxisalltags.

Deklaration von Helsinki? CIOMS? VKlin? Dies alles musste ich «googeln». Mit meiner Ignoranz stehe ich nicht alleine da, das wurde mir im Gespräch mit befreundeten Ärzten bald klar. Dass viele klinische Versuche nicht direkt von Teams der Pharmafirmen durchgeführt werden, sondern an «Contract Research Organizations» (CRO) delegiert werden, war mir nicht bewusst, ebenso wenig, dass etwa die Hälfte der klinischen Versuche in Ländern vorgenommen wird, in denen grosse Armut herrscht und/ oder Menschenrechte missachtet werden, wie in Russland, China, Mexico, Argentinien usw.

Weshalb werden immer mehr Versuche in diese Länder verlagert?

\section{«Mit meiner Ignoranz stehe ich nicht alleine da.»}

Bei der Einführung eines neuen Medikaments entfallen gemäss Angaben des Dachverbandes der Pharmafirmen scienceINDUSTRIES $2 / 3$ der Kosten auf die klinischen Versuche. Je schneller diese Phase abgeschlossen werden kann, umso länger dauert die patentgeschützte lukrative Vertriebszeit.

Die EvB nennt folgende Gründe, welche die oben erwähnten Länder für Pharmafirmen als Versuchsfeld attraktiv machen: billige Prüfinstanzen, kulante Bewilligungsverfahren, «entgegenkommende» Ethikkommissionen und ein erheblich schnelleres Anwerben von Testpersonen aus armen und ungebildeten Schichten. Schadenersatzforderungen seien zudem von Menschen aus diesen Gebieten kaum zu erwarten.

Vertreter der Pharmaindustrie betonen die Notwendigkeit, die Einhaltung der kodifizierten ethischen Normen zu kontrollieren, um Missbräuchen vorzubeugen. Die werde durch die Anstellung von «Clinical Research Associates» als «Monitor» gewährleistet, die in Problemländern mit der Supervision der klinischen Versuche betraut werden (und von den «Sponsoren» bezahlt sind). Ferner würden regelmässige Audits durchgeführt. Im Schlussrapport werde auch über die Einhaltung der ethischen Normen Bericht erstattet (wobei der Rapport intern bleibt).

Für die Durchführung von Versuchen in Schwellenländern gibt die Pharmaindustrie andere Gründe an als die EvB: Primär gehe es nicht um Kosteneinsparungen, sondern darum, Versuchspersonen aus verschiedenen Ethnien und Kulturen zu testen in verschiedenen Ländern, z.B. China, Russland, Indien, Äthiopien oder Taiwan. Zudem würden für eine Marktzulassung oft lokal durchgeführte Studien verlangt. Ferner spiele die Prävalenz diverser Krankheiten eine Rolle, z. B. im Falle von Malaria.

Die «Erklärung von Bern» fordert, dass die Swissmedic die Einhaltung der ethischen Grundsätze im Sinn der «Deklaration von Helsinki» überprüft, bevor ein Medikament in der Schweiz zugelassen wird. Dies würde aber voraussetzen, dass Versuchsprotokolle und wahrheitsgetreue Berichte des «Monitors» der Swissmedic vorliegen.

Der immer wieder bekräftigte Wille der Pharmafirmen, die Einhaltung ihres ethischen Kodex' intern durchzusetzen und Transparenz zu schaffen, ist zwar zu begrüssen, trotzdem müssen sie auch eine Supervision durch unabhängige Instanzen akzeptieren und der Swissmedic und der Öffentlichkeit gegenüber Rechenschaft ablegen.

Es ist wichtig, dass sie ihre ethische Verpflichtung in einfacher Sprache ausformuliert und gut zugänglich macht.

Als Diskussionsanstoss möchte ich einige Punkte erwähnen, die zum Teil in den firmeneigenen Verpflichtungen und in diversen internationalen Codices verstreut enthalten sind:

- Alle klinischen Studien müssen zentral angemeldet und registriert sowie fortlaufend nummeriert werden. Die Daten aller einmal begonnenen Stu- 
dien müssen vollständig und transparent den zuständigen Arzneimittelbehörden (Swissmedic, EMA, FDA usw.) vorgelegt werden.

- Die Studienergebnisse müssen integral veröffentlicht werden. Nach Markteinführung sollen sie öffentlich zugänglich sein.

\section{«Billige Prüfinstanzen, kulante Bewilligungsverfahren, <entgegenkommende〉 Ethikkommissionen ...»}

- Fälschungen, Verheimlichung negativer Daten, Unterschlagung ganzer Versuchssequenzen müssen mit empfindlichen Sanktionen belegt und strafrechtlich verfolgt werden. «Whistleblower» dürfen keine persönlichen Nachteile erleiden.

- Die «Sponsoren» müssen gewährleisten, dass die zuständigen Ethikkommissionen kompetent besetzt sind und korrekt arbeiten, besonders in Ländern, in denen eine staatliche Aufsicht ungenügend gewährleistet ist. Dort müssen die Firmen selber kontrollieren, dass die Arbeit der Ethikkommissionen und die Durchführung der klinischen Versuche der international anerkannten «Good Clinical Practice» GCP entspricht. Ein entsprechender Schlussbericht ist der Swissmedic (resp. den anderen Arzneimittelbehörden) vorzulegen.

- Allen Versuchspersonen muss in ihrer Sprache der Versuchsablauf präzise erklärt und ein gutverständlicher Vertrag abgeschlossen werden. Den Versuchspersonen muss eine Ombudsstelle und ein Rechtsweg für Beschwerden bekannt und zugänglich sein.

- Menschen aus armen Gegenden, die im Rahmen eines klinischen Versuche vielleicht erstmals eine adäquate Behandlung erfahren haben, darf man später nicht fallen lassen.

- "Pharmaceutical companies should adhere to both the spirit and the letter of applicable industry codes. To achieve this, pharmaceutical companies will ensure that all relevant personnel are appropriately trained.» (aus der Präambel der internationalen Vereinigung der Pharmaindustrie IFPMA)

Wollen Sie diesen Artikel kommentieren? Nutzen Sie dafür die Kommentarfunktion in der OnlineVersion oder sehen Sie nach, was Ihre Kolleginnen und Kollegen bereits geschrieben haben: www.saez.ch/ aktuelle-ausgabe/ interaktive-beitraege/ ethische Prinzipien aufrechtz Schweizer Pharmaindustrie und Kontrollen sind zwar notwendig und nützlich, können aber Respekt und menschliches Mitgefühl nicht ersetzen. Haben wir den Mut, uns zu fragen, ob wir selbst an diesem Versuch mitmachen würden, um der Menschheit einen Dienst zu erweisen? Würden wir den Versuch an unseren Kindern erlauben?
Good Clinical Practice konsequent zu pflegen, braucht Energie und kostet Geld. Es ist auch eine Frage der Intelligenz.

Auf lange Frist ist GCP eine hervorragende Investition, vielleicht die beste!

Es ist nämlich erwiesen, dass die ethische Kultur einer Firma Gold wert ist, denn damit wird bei der Belegschaft die Identifikation mit den Firmenzielen und ein engagiertes Mitdenken gefördert. Es kommt nachweislich zu weniger Absenzen und Krankheitsfällen, und die Kooperationsbereitschaft innerhalb der Firma verstärkt sich. Eine klare ethische Haltung ist für das Renommee von unschätzbarem Wert, exorbitante finanzielle «Incentives» hingegen schüren mehr Begehrlichkeiten, fördern firmeninterne Konkurrenz und Intrigen, treiben Manager ins Burnout und demoralisieren die Belegschaft der unteren Etagen, die Basis jeden Betriebserfolges.

Es geht um das Gleichgewicht der Werte. Aktionäre wollen (meist) Geld und einen hohen Börsenkurs sehen, was in Grenzen legitim ist. Kunden, andere «Stakeholders» und die breite Öffentlichkeit hingegen sind am Produkt interessiert: qualitativ gute, sorgfältig und ethisch korrekt geprüfte Medikamente.

\section{«Die Würde der Versuchsperson}

\section{muss dabei absolut unantastbar}

\section{bleiben.»}

Beide Werte wollen gepflegt sein. Die Würde der Versuchsperson muss dabei absolut unantastbar bleiben. «Fair Pharma» müsste eigentlich eine Selbstverständlichkeit sein, oder braucht es auch hier ein spezielles Label?

Mit ihrer Kampagne hat die EvB einen mächtigen Pflasterstein in den Teich geworfen, dessen Wellenschlag nicht so schnell verebben wird. Es ist zu hoffen, dass es nicht zu einem sterilen Hickhack von Schuldzuweisungen und Dementis kommt, sondern dass «proaktiv», im Sinne der Verantwortungsethik (Max Weber), die Firmenkultur der Pharmaindustrie gestärkt aus dieser kalten Dusche hervorgeht, und die Leiter der Pharmafirmen in Zukunft noch intensiver dafür sorgen, dass die ethischen Richtlinien der «Deklaration von Helsinki» strikt eingehalten werden.

Wir Ärztinnen und Ärzte sind ebenfalls aufgefordert, die Augen zu öffnen und, wenn nötig, den Mund!

Der Autor freut sich auf Kritik, Ergänzungen, Vorschläge und Präzisierungen, besonders vonseiten der pharmazeutischen Industrie.

Literatur auf Anfrage. Das meiste kann einfach gegoogelt werden. 